[2] S. Boyd and C. Barratt, Linear Controller Design-Limits of Performance. Upper Saddle River, NJ: Prentice-Hall, 1991.

[3] G. C. Calafiore, F. Dabbene, and R. Tempo, "Randomized algorithms for probabilistic robustness with real and complex structured uncertainty," IEEE Trans.Automat. Contr., vol. 45, pp. 2218-2235, Dec. 2000

[4] G. C. Calafiore and F. Dabbene, "Randomization in $R H_{\infty}$ : An approach to control design with hard/soft performance specications," in Proc. 41 st IEEE Conf. Decision Control, Las Vegas, NV, 2002, pp. 2260-2265.

[5] G. Calafiore, F. Dabbene, and R. Tempo, "Uniform sample generation in $l_{p}$ balls for probabilistic robustness analysis," in Proc. 37th IEEE Conf. Decision Control, Tampa, FL, 1998, pp. 3335-3340.

[6] G. Calafiore and B. T. Polyak, "Stochastic algorithms for exact and approximate feasibility of robust LMI's," IEEE Trans. Automat. Contr. vol. 46, pp. 1755-1759, Nov. 2001.

[7] X. Chen and K. Zhou, "A probabilistic approach to robust control," in Proc. 36th IEEE Conf. Decision Control, San Diego, CA, 1997, pp. 4894-4895.

[8] —, "Constrained optimal synthesis and robustness analysis by randomized algorithms," in Proc. 1998 Amer. Control Conf., vol. 3, Philadelphia, PA, 1998, pp. 1429-1433.

[9] L. Devroye, "Random variate generation for multivariate unimodal densities," ACM Trans. Modeling Comput. Simul., vol. 7, pp. 447-477, 1997.

[10] Y. Fujisaki, F. Dabbene, and R. Tempo, "Probabilistic robust design of LPV control systems," in Proc. 40th IEEE Conf. Decision Control, Orlando, FL, 2001, pp. 2019-2024.

[11] C. M. Lagoa, M. Sznaier, and B. R. Barmish, "An algorithm for generating transfer functions uniformly distributed over $H_{\infty}$ balls," in Proc. 40th IEEE Conf. Decision Control, Orlando, FL, 2001, pp. $5038-5043$.

[12] C. I. Marrison and R. F. Stengel, "Stochastic robustness synthesis applied to a benchmark control problem," Int. J. Robust Nonlinear Control, vol. 5, no. 1, pp. 13-31, 1995.

[13] _ "Robust control system design using random search and genetic algorithms," IEEE Trans. Automat. Contr., vol. 42, pp. 835-839, June 1997.

[14] T. Motoda, R. F. Stengel, and Y. Miyazawa, "Robust control system design using simulated annealing," J. Guid., Control, Dyna., vol. 25, no. 2, pp. 267-274, Mar. 2002.

[15] B. T. Polyak and R. Tempo, "Probabilistic robust design with linear quadratic regulators," in Proc. 39th IEEE Conf. Decision Control, Sydney, NSW, Australia, 2000, pp. 1037-1042.

[16] L. R. Ray and R. F. Stengel, "A Monte Carlo approach to the analysis of control system robustness," Automatica, vol. 29, no. 1, pp. 229-236, 1993.

[17] R. S. Sánchez-Peña and M. Sznaier, Robust Systems: Theory and Applications. New York: Wiley, 1998.

[18] R. F. Stengel and L. E. Ryan, "Stochastic robustness of linear timeinvariant control systems," IEEE Trans. Automat. Contr., vol. 36, pp. 82-87, Jan. 1991

[19] Q. Wang and R. F. Stengel, "Robust control of nonlinear systems with parametric uncertainty," Automatica, vol. 38, no. 9, pp. 1591-1599, September 2002.

[20] D. Williams, Probability With Martingales. New York: Cambridge Univ. Press, 1991.

[21] A. Yoon, P. Khargonekar, and K. Hebbale, "Design of computer experiments for open-loop control and robustness analysis of clutch-to-clutch shifts in automatic transmissions," in Proc. 1997 Amer. Control Conf., vol. 5, Albuquerque, NM, 1997, pp. 3359-3364.

[22] A. Yoon and P. Khargonekar, "Computational experiments in robust stability analysis," in Proc. 36th IEEE Conf. Decision Control, San Diego, CA, 1997, pp. 3260-3265.

\section{Invariant Subspaces for LPV Systems and Their Applications}

\author{
Gary Balas, József Bokor, and Zoltán Szabó
}

\begin{abstract}
The aim of this note is to extend the notion of invariant subspaces known in the geometric control theory of the linear time invariant systems to the linear parameter-varying (LPV) systems by introducing the concept of parameter-varying invariant subspaces. For LPV systems affine in their parameters, algorithms are given to compute many parameter varying subspaces relevant in the solution of state feedback and observer design problems.
\end{abstract}

Index Terms-Distributions, input affine systems, invariant subspaces, linear parameter-varying (LPV) systems.

\section{INTRODUCTION}

Important engineering processes involve time-varying linear and nonlinear models. A general theory for the robust control of nonlinear systems is not computationally tractable and useful progress requires an intermediate level of complexity. Linear parameter-varying (LPV) modeling techniques have gained a lot of interest as they provide a systematic means of computing gain-scheduled controllers, especially those related to vehicle and aerospace control, [2], [4], [11], [18], [23].

Many of the control system design techniques using LPV models can be cast or recast as convex feasibility problem with infinite constraints that involve linear matrix inequalities (LMIs). This problem can be addressed by using affine LPV modeling that reduces the infinite constraints imposed on the LMI formulation to a finite number, [1], [30].

The pure LPV model is not quite matched for practical problems, e.g., to the flight control problem, where the scheduling variables are in fact system states (e.g. airspeed and angle of attack), rather than bounded external variables. An approach to this problem is to generate so-called quasi-LPV models, which are applicable when the scheduling variables are measured states, the dynamics are linear in the inputs and other states, and there exist inputs to regulate the scheduling variables to arbitrary equilibrium values.

The mathematically dual concepts of $(\mathrm{A}, \mathrm{B})$ (or controlled)-invariance and (C,A) (or conditioned)-invariance play an important role in the geometric theory of linear time-invariant (LTI) systems, [6], [32]. These concepts were used to study some fundamental problems of LTI control theory, such as disturbance decoupling (DDP), unknown input observer design, fault detection (FPRG), [19], [20], [32]. The nonlinear version of this geometrical approach is much more complex and deals with certain locally controlled or conditioned invariant distributions and codistributions, [14], [15], [24], [25]

The aim of this note is to extend these notions for the parameter-varying systems by introducing the notion of parameter-varying $(A, B)$-invariant, parameter-varying $(C, A)$-invariant, controllability and unobservability subspaces, and to give some algorithms to compute these subspaces if certain conditions are fulfilled.

Manuscript received November 19, 2002; revised July 22, 2003. Recommended by Associate Editor P. A. Iglesias. This work was supported in part by NASA Langley under NASA Grant NCC-1-337 (Dr. C. M. Belcastro Technical Monitor) and in part by the Hungarian National Science Foundation (OTKA) under Grant T 030182

G. Balas is with the Department of Aerospace Engineering and Mechanics, University of Minnesota, Minneapolis, MN 55455 USA

J. Bokor and Z. Szabó are with the Computer and Automation Research Institute, Hungarian Academy of Sciences, H-1111 Budapest, Hungary (e-mail: bokor@sztaki.hu; szaboz@decst.scl.sztaki.hu).

Digital Object Identifier 10.1109/TAC.2003.819647 
This note deals with the class of LPV systems of $m$ inputs and $p$ outputs that can be described as

$$
\begin{aligned}
\dot{x}(t) & =A(\rho(t)) x(t)+B(\rho(t)) u(t) \\
y(t) & =C(\rho(t)) x(t)
\end{aligned}
$$

where

$$
\begin{aligned}
& A(\rho(t))=A_{0}+\rho_{1}(t) A_{1}+\cdots+\rho_{N}(t) A_{N} \\
& B(\rho(t))=B_{0}+\rho_{1}(t) B_{1}+\cdots+\rho_{N}(t) B_{N} \\
& C(\rho(t))=C_{0}+\rho_{1}(t) C_{1}+\cdots+\rho_{N}(t) C_{N}
\end{aligned}
$$

and the dimension of the state space is supposed to be $n$.

It is assumed that each parameter $\rho_{i}$ ranges between known external values $\rho_{i}(t) \in\left[\rho_{i}, \bar{\rho}_{i}\right]$ and the parameter set, that contains all the vectors $\rho:=\left[\rho_{1}(t), \ldots, \rho_{N}(t)\right]^{T}$ where $t \in[0, T]$, will be denoted by $\mathcal{P}$. For the sake of notational simplicity the time dependency of the matrices will be omitted $(A(\rho):=A(\rho(t)))$ where it is possible.

In introducing the various parameter-varying invariant subspaces, an important goal was to set notions that lead to computationally tractable algorithms. In general, it is a hard task to give an exhaustive characterization for the solution of the fundamental problems such as the DDP or the FPRG problem even in the LPV case. However, since the main ingredient in the solution of these problems are certain local decomposition theorems - in observable and unobservable subsystems, for example-using suitable invariant subspaces instead of the distributions or codistributions one can get sufficient conditions for the solvability that can be useful for practical engineering problems.

\section{CONTROLlability AND OBSERVABILITY OF LiNEAR SySTEMS}

In what follows some standard results, see, e.g. [17] and [26], will be recalled about the controllability and observability of the LTV systems:

$$
\begin{aligned}
& \dot{x}(t)=A(t) x(t)+B(t) u(t) \\
& y(t)=C(t) x(t)
\end{aligned}
$$

where $x(t) \in \mathcal{X} \subset \mathbb{R}^{n}, x_{0}=x\left(t_{0}\right), u(t) \in \mathbb{R}^{m}$ and $y(t) \in \mathbb{R}^{p}$.

A convenient way to study all solutions of a linear equation on the interval $\left[t_{0}, T\right]$, for all possible initial values simultaneously, is to introduce the matrix differential equation

$$
\dot{X}(t)=A(t) X(t) \quad X\left(t_{0}\right)=\rrbracket
$$

with $X(t) \in \mathbb{R}^{n \times n}$. Let us denote by $\Phi\left(t, t_{0}\right)$ the transition matrix, i.e., the solution of (7) at time $t$; it is known that $\Phi\left(t, t_{0}\right)$ is nonsingular for any $t$.

Definition 1: A state $x_{0}$ is said to be controllable at time $t_{0}$ if there exist a control function $u(t)$ depending on $x_{0}$ and $t_{0}$ and defined over some finite closed interval $\left[t_{0}, T\right]$ such that for the corresponding solution one has $x(T)=\phi_{x_{0}}\left(T, t_{0}\right)=0$. If this is true for every state $x$ and every $t_{0}$ then the system will be called (completely) controllable.

System (5) is called (completely) observable on an interval $\left[t_{0}, T\right]$ if any initial state $x_{0}$ at $t_{0}$ can be determined from knowledge of the system output $y(t)$ and input $u(t)$ over the interval $\left[t_{0}, T\right]$.

For a given system (5) let us denote by $\mathcal{R}$ the reachability subspace, i.e., the set of all states that can be reached from the origin in any finite time by means of control actions. The system is controllable if and only if $\mathcal{R}=\mathcal{X}$.

Analogously let us denote by $\mathcal{Q}$ the unobservability subspace, i.e., the set of all initial states that cannot be recognized from the output function. The system is observable if and only if $\mathcal{Q}=0$.

One of the fundamental results, see [17], concerning controllability and observability properties of LTV systems uses the system solution in the formulation of its conditions. The solution of such a system is generally not available in closed form. It is also a standard result that one can derive a rank condition that guarantees controllability which does not involve integration and can be obtain directly from the data $(A(t), B(t))$, see [26]:

Proposition 1: If (5) is analytic on an interval $\mathcal{I}$ and $t$ is an arbitrary fixed element of $\mathcal{I}$, then (5) is completely controllable on every nontrivial subinterval of $\mathcal{I}$ if and only if

$$
\operatorname{rank}\left[B_{0}(t) \quad B_{1}(t) \quad \cdots \quad B_{k}(t)\right]=n
$$

for some integer $k$, where

$$
B_{0}(t):=B(t) \quad B_{i+1}(t):=A(t) B_{i}(t)-\frac{d}{d t} B_{i}(t) .
$$

If the analyticity condition is dropped, then the rank condition is only sufficient. An analogous result holds for observability, too.

It is also a standard way to study questions concerning controllability and observability using the Volterra expansions associated to the given differential equations. If the time-varying system has an affine structure then one can exploit this structure in deciding controllability and observability questions. Using the Peano-Baker formula for the transition matrix, one can prove the following result.

Lemma 1: For (1), the points attainable from the origin are those from the subspace spanned by the vectors

$$
\mathcal{R}_{(\mathcal{A}, \mathcal{B})}:=\operatorname{span}\left\{\prod_{j=1}^{K} A_{l_{j}}^{i_{j}} B_{k}\right\}
$$

where $K \geq 0, l_{j}, k \in\{0, \cdots, N\}, i_{j} \in\{0, \cdots, n-1\}$. Moreover, if one consider the finitely generated Lie-algebra $\mathcal{L}\left(A_{0}, \ldots, A_{N}\right)$ which contains $A_{0}, \ldots, A_{N}$, and a basis $\hat{A}_{1}, \ldots, \hat{A}_{K}$ of this algebra, then

$$
\mathcal{R}_{(\mathcal{A}, \mathcal{B})}=\sum_{l=0}^{N} \sum_{n_{1}=0}^{n-1} \ldots \sum_{n_{K}=0}^{n-1} \operatorname{Im}\left(\hat{A}_{1}^{n_{1}} \ldots \hat{A}_{K}^{n_{K}} B_{l}\right) .
$$

A direct consequence of this fact is that if the inclusion $\mathcal{R}_{\mathcal{A}, \mathcal{B}} \subset \mathbb{R}^{n}$ is strict, i.e, if $\mathcal{R}_{\mathcal{A}, \mathcal{B}}$ is a proper subspace, then the system (5) cannot be completely controllable.

The main question is that under which condition is controllability guaranteed if the relation $\mathcal{R}_{\mathcal{A}, \mathcal{B}}=\mathbb{R}^{n}$, i.e., the multivaraiable Kalman rank condition, holds. In [28] and [29], an answer was given in terms of the solutions of a Wei-Normann equation, however, that solution is seldom accessible in practical situations. In [29], it was shown that if the parameter functions $\left\{1, \rho_{1}, \cdots, \rho_{N}\right\}$ are differential algebraically independent (relation that also involves the derivatives of $\rho_{i}$ ), then the multivaraiable Kalman rank condition is also sufficient for controllability. An analogous result holds for observability, as well.

\section{PARAMETER-VARYING INVARIANT SUBSPACES}

For LTI systems the concept of certain invariant subspaces and the corresponding global decompositions of the state equations induced by these invariant subspaces was one of the main thrusts for the development of geometric methods for solutions to problems of disturbance decoupling or noninteracting control; see [32]. Nonlinear systems can be studied using tools from differential geometry, when the central role is played by the concept of invariant distributions. From the geometric viewpoint results of the classical linear control can be seen as special cases of more general nonlinear results; for details, see [15] and [21].

Due to the computational complexity involved, these nonlinear methods have limited applicability in practice.

Linear time varying systems can be viewed as affine nonlinear systems [14], by augmenting the original state space to $\xi:=[t, x]^{T}$. Restricting the investigations to linear subspaces, as special instances of distributions, then a subspace $\mathcal{V}$ of $\mathbb{R}^{n}$, will be an invariant distribution for system (5) if and only if $A(\rho(t)) \mathcal{V} \subset \mathcal{V}$ for all $t \in \mathcal{I}$, where $\mathcal{I}$ is an interval on which the solutions are defined.

This fact motivates the introduction of the following notion for LPV systems. 
Definition 2: A subspace $\mathcal{V}$ is called parameter-varying invariant subspace for the family of the linear maps $A(\rho)$ (or shortly $\mathcal{A}$-invariant subspace) if

$$
A(\rho) \mathcal{V} \subset \mathcal{V} \text { for all } \rho \in \mathcal{P} \text {, i.e., for all } t \in \mathcal{I} .
$$

Let us observe, that if $\mathcal{V}$ is an $\mathcal{A}$-invariant subspace and $\operatorname{Im} B(t) \subset$ $\mathcal{V}$ for all $t$, then, considering the restrictions of the state matrices to this subspace, i.e.,

$$
\bar{A}(\rho):=A(\rho) \mid \mathcal{V}
$$

and using the invariancy property, (5) can be decomposed as

$$
\begin{aligned}
& \dot{\bar{x}}(t)=\bar{A}(t) \bar{x}(t)+\tilde{A}_{1}(t) \tilde{x}(t)+\bar{B}(t) u(t) \\
& \dot{\tilde{x}}(t)=\tilde{A}_{2}(t) \tilde{x}(t) .
\end{aligned}
$$

It is obvious that the subspace $\mathcal{R}_{(\mathcal{A}, \mathcal{B})}$ in (10) is $A(\rho(t))$ invariant, i.e.,

$$
A(\rho(t)) \mathcal{R}_{(\mathcal{A}, \mathcal{B})} \subseteq \mathcal{R}_{(\mathcal{A}, \mathcal{B})}, \quad \text { for all } t
$$

moreover, one has that for the induced decomposition

$$
\mathcal{R}_{(\mathcal{A}, \mathcal{B})}=\mathcal{R}_{(\overline{\mathcal{A}}, \overline{\mathcal{B}})} .
$$

These facts motivate the introduction of the following notion.

Definition 3: Let $\mathcal{B}(\rho)$ denote $\operatorname{Im} B(\rho)$. Then a subspace $\mathcal{V}$ is called a parameter-varying $(\mathrm{A}, \mathrm{B})$-invariant subspace (or shortly $(\mathcal{A}, \mathcal{B})$-invariant subspace) if for all $\rho \in \mathcal{P}$ any of the following equivalent conditions holds:

$$
\text { a.) } A(\rho) \mathcal{V} \subset \mathcal{V}+\mathcal{B}(\rho)
$$

and there exists a mapping $F \circ \rho:[0, T] \rightarrow \mathbb{R}^{m \times n}$ such that

$$
\text { b.) }(A(\rho)+B(\rho) F(\rho)) \mathcal{V} \subset \mathcal{V} \text {. }
$$

Dealing with parametric uncertainties a similar concept was introduced in [7], called robust controlled invariant subspace. If one sets the gain matrix to be constant then the resulting subspace will be more restrictive, this approach was used in [9] and [22], and was termed as generalized controllability $(A, B)$-invariant subspace.

The dual notion of the previous definition is the following.

Definition 4: Let $\mathcal{C}(\rho)$ denote $\operatorname{Ker} C(\rho)$. Then a subspace $\mathcal{W}$ is called a parameter-varying (C,A)-invariant subspace (or shortly $(\mathcal{C}$, $\mathcal{A}$ )-invariant subspace) if for all $\rho \in \mathcal{P}$ any of the following equivalent conditions holds:

$$
\text { a.) } \quad A(\rho)(\mathcal{W} \cap \mathcal{C}(\rho)) \subset \mathcal{W}
$$

and there exists a mapping $G \circ \rho:[0, T] \rightarrow \mathbb{R}^{n \times p}$ such that:

$$
\text { b.) }(A(\rho)+G(\rho) C(\rho)) \mathcal{W} \subset \mathcal{W} \text {. }
$$

The mathematically dual concepts of (A,B) (or controlled)-invariance and (C,A) (or conditioned)-invariance play an important role in the geometric theory of LTI systems. These concepts were used to study some fundamental problems of LTI control theory, e.g., [6], [19], [20], and [32].

In the time-varying case, one can deal with these problems using the much more complex mathematical objects given by the locally controlled or conditioned invariant distribution algorithms or codistribution algorithms, respectively, [15], [24]. The main problem that arises in practical situations is that either one cannot perform the computations or one cannot verify the conditions under the given algorithms provide the desired results.

If certain conditions are fulfilled, e.g., if the parameter functions are differential algebraically independent, then the parameter invariant subspaces defined above coincide with the corresponding invariant distribution or codistribution, respectively. However, to give sufficient conditions for the solution of certain state feedback and observer filter design problems it is enough that some decompositions of the state equations could be performed. The parameter-varying versions of these invariant spaces are suitable objects to define the required decompositions, therefore they can play the same role in the solution of the fundamental problems, such as disturbance decoupling (DDP), see [31], unknown input observer design, fault detection (FPRG), see [3], [5], [27], as their counterparts in the time invariant context.

\section{INVARIANCE ALGORITHMS}

In [10], an algorithm was given to determine the robust controlled invariant subspace, however since the number of conditions is not finite, the algorithm, in general, is quite complex. From a practical point of view, it is an important question to characterize these parameter-varying subspaces by a finite number of conditions. Assuming the special structure of the matrix $A(\rho)$ in (1), it is immediate that if the inclusions hold for all $A_{i}$, then they hold also for all $\rho \in \mathcal{P}$. It is not so straightforward under which conditions the reverse implication is true, too.

In what follows, a sufficient condition will be given that characterizes property (11) using only a finite number of constrains.

Lemma 2: If the functions $1, \rho_{1}, \ldots, \rho_{N}$ are linearly independent over $\mathbb{R}$ then $A(\rho) \mathcal{V} \subset \mathcal{W} \forall \rho \in \mathcal{P}$ if and only if

$$
A_{i} \mathcal{V} \subset \mathcal{W}, \quad i=0, \ldots, N .
$$

We are interested in finding supremal $\mathcal{A}$-invariant subspaces in a given subspace $\mathcal{K}$ or containing a given subspace $\mathcal{L}$. As far as the first purpose is concerned the $\mathcal{A}-\mathcal{I}$ nvariant $\mathcal{S}$ ubspace $\mathcal{A l g o r i t h m}$ over $\mathcal{L}$ can be defined as:

$$
\begin{aligned}
\mathcal{A} \mathcal{I} \mathcal{A L}: \mathcal{V}_{0} & =\mathcal{L} \\
\mathcal{V}_{k+1} & =\mathcal{L}+\sum_{i=0}^{N} A_{i} \mathcal{V}_{k}, \quad k \geq 0 \\
\mathcal{V}^{*} & =\lim _{k \rightarrow \infty} \mathcal{V}_{k} .
\end{aligned}
$$

Obviously, the algorithm will stop after a finite number of steps, i.e., $\mathcal{V}^{*}=\mathcal{V}_{n-1}$.

Theorem 1: The subspace $\mathcal{V}^{*}$ given by (21) is such that

$$
\begin{aligned}
& \mathcal{L} \subset \mathcal{V}^{*} \\
& \mathcal{V}^{*} \text { is } \mathcal{A} \text { - invariant }
\end{aligned}
$$

and assuming that conditions of Lemma 2. hold, it is minimal with these properties.

Similar to the linear case the subspace $\mathcal{V}^{*}$ will be denoted by $\langle\mathcal{A} \mid \mathcal{L}\rangle$. By duality, one has the $\mathcal{A}-\mathcal{I}$ nvariant $\mathcal{S}$ ubspace $\mathcal{A}$ lgorithm in $\mathcal{K}$, i.e.,

$$
\begin{aligned}
\mathcal{A I S} \mathcal{A K}: \mathcal{W}_{0} & =\mathcal{K} \\
\mathcal{W}_{k+1} & =\mathcal{K} \cap \bigcap_{i=0}^{N} A_{i}^{-1} \mathcal{W}_{k}, \quad k \geq 0 \\
\mathcal{W}^{*} & =\lim _{k \rightarrow \infty} \mathcal{W}_{k}
\end{aligned}
$$

where $A_{i}^{-1} \mathcal{W}_{k}$ denotes the inverse image of $\mathcal{W}_{k}$ under $A_{i}^{-1}$. The subspace $\mathcal{W}^{*}$ will be denoted by $\langle\mathcal{K} \mid \mathcal{A}\rangle$.

Theorem 2: The subspace $\mathcal{W}^{*}$ given by (24) is such that

$$
\begin{aligned}
& \mathcal{W}^{*} \subset \mathcal{K} \\
& \mathcal{W}^{*} \text { is } \mathcal{A} \text { - invariant }
\end{aligned}
$$

and assuming that that conditions of Lemma lem:pc. holds, it is maximal with these properties. 
The direct application of nonlinear algorithms to LPV systems yield time-dependent distributions. In order to get rid of the time-dependency, we will continue with the following argument. The set of all $(\mathcal{A}, \mathcal{B})$-invariant subspaces contained in a given subspace $\mathcal{K}$, is an upper semilattice with respect to subspace addition. This semilattice admits a maximum which can be computed from the $(\mathcal{A}, \mathcal{B})$ - $\mathcal{I}$ nvariant $\mathcal{S}$ ubspace Algorithm:

$$
\begin{aligned}
\mathcal{A B I S A}: \quad \mathcal{V}_{0} & =\mathcal{K} \\
\mathcal{V}_{k+1} & =\mathcal{K} \cap \bigcap_{i=0}^{N} A_{i}^{-1}\left(\mathcal{V}_{k}+\mathcal{B}\right) .
\end{aligned}
$$

The limit of this algorithm will be denoted by $\mathcal{V}^{*}$ and its calculation needs at most $n$ steps.

The set of all $(\mathcal{C}, \mathcal{A})$-invariant subspaces-note that $\mathcal{C}=$ $\mathrm{Ker} C$-containing a given subspace $\mathcal{L}$, is a lower semilattice with respect to subspace intersection. This semilattice admits a minimum which can be computed from the $(\mathcal{C}, \mathcal{A})$ - $\mathcal{I}$ nvariant $\mathcal{S}$ ubspace $\mathcal{A l g o r i t h m}$ that can be obtained by duality from the $\mathcal{A B I S \mathcal { A }}$ algorithm, see [27].

These algorithms can be obtained in the same way as those for the LTI case; see [32]. The minimality (maximality) of these subspaces follows from the fact that if one assumes that the parameter functions are linearly independent, then any relation involving subspace inclusions of the type $A(\rho) \mathcal{V} \subset \mathcal{W}$ will be equivalent with the same type of inclusions with the matrices $A_{i}$, i.e., $A_{i} \mathcal{V} \subset \mathcal{W}$ for all $i$.

For the LPV case (with constant $B$ matrix) one can get the following definition for the controllability subspace.

Definition 5: A subspace $\mathcal{R}$ is called parameter-varying controllability subspace if there exists a constant matrix $K$ and a parameter varying matrix $F:[0, T] \rightarrow \mathbb{R}^{m \times n}$ such that

$$
\mathcal{R}=\langle\mathcal{A}+B \mathcal{F} \mid \operatorname{Im} B K\rangle
$$

where the notation $\mathcal{A}+B \mathcal{F}$ stems for the system $A(\rho)+B F(\rho)$.

As in the classical case, it can be seen that the family of controllability subspaces contained in a given subspace $\mathcal{K}$ is closed under subspace addition. Hence, this family has a maximal element which can be computed from the parameter-varying $\mathcal{C}$ ontrollability $\mathcal{S}$ ubspace Algorithm:

$$
\begin{aligned}
\mathcal{C S} \mathcal{A}: \mathcal{R}_{0} & =0 \\
\mathcal{R}_{k+1} & =\mathcal{V}^{*} \cap\left(\sum_{i=0}^{N} A_{i} \mathcal{R}_{k}+\mathcal{B}\right) \\
\mathcal{R}^{*} & =\lim _{k \rightarrow \infty} \mathcal{R}_{k}
\end{aligned}
$$

where $\mathcal{V}^{*}$ is computed by $\mathcal{A B I S} \mathcal{A}$.

Theorem 3: The subspace $\mathcal{R}^{*}$ is the largest parameter-varying controllability subspace in $\mathcal{C}$.

This subspace plays a central role in solving the DDP problem in the affine LPV case; for details, see [31].

The $\mathcal{C S} \mathcal{A}$ algorithm can be obtained by dualizing the observability codistribution algorithm, see [24], and applying it for subspaces.

In what follows, a few properties of the controllability subspaces, known in the LTI case, [32], will be recast in the parameter varying context.

Theorem 4: If $\hat{\mathcal{B}} \subset \mathcal{B}$ and $\mathcal{R}=\langle\mathcal{A} \mid \mathcal{B}\rangle$ then $\mathcal{R}=\langle\mathcal{A} \mid \mathcal{B} \cap \mathcal{R}\rangle$. Conversely, if $\mathcal{R}=\langle\mathcal{A} \mid \mathcal{B} \cap \mathcal{R}\rangle$ then there exists $K: \mathcal{U} \rightarrow \mathcal{U}$ for which $\mathcal{R}=\langle\mathcal{A} \mid \operatorname{Im} B K\rangle$.

Theorem 5: A subspace $\mathcal{R}$ is parameter-varying controllability subspaces if and only if there exists $F:[0, T] \rightarrow \mathbb{R}^{m \times n}$ such that $\mathcal{R}=$ $\langle\mathcal{A}+B \mathcal{F} \mid \mathcal{B} \cap \mathcal{R}\rangle$.

Let $\mathcal{R} \subset \mathcal{X}$ a fixed subspace and define $\Gamma=\{\mathcal{Z}: \mathcal{Z}=\mathcal{R} \cap$ $\left.\left(\sum_{i=0}^{N} A_{i} \mathcal{Z}+\mathcal{B}\right)\right\}$.
Lemma 3: There exists a unique minimal element of $\Gamma$.

The minimal element $\mathcal{Z}^{*}$ can be computed from the following algorithm:

$$
\mathcal{Z}_{0}=0 \quad \mathcal{Z}_{k+1}=\mathcal{R} \cap\left(\sum_{i=0}^{N} A_{i} \mathcal{S}_{k}+\mathcal{B}\right) .
$$

Theorem 6: A subspace $\mathcal{R} \subset \mathcal{X}$ is parameter-varying controllability subspace if and only if it is $(\mathcal{A}, \mathcal{B})$-invariant and $\mathcal{R}=\mathcal{Z}^{*}$.

The dual notion of parameter-varying controllability subspace is the following:

Definition 6: A subspace $\mathcal{S}$ is called parameter-varying unobservability subspace if there exists a constant matrix $H$ and a parameter varying matrix $G:[0, T] \rightarrow \mathbb{R}^{n \times p}$ such that

$$
\mathcal{S}=\langle\operatorname{Ker} H C \mid \mathcal{A}+\mathcal{G} C\rangle
$$

where $\mathcal{A}+\mathcal{G} C$ denotes the system $A(\rho)+G(\rho) C$.

By dualization one can show that the family of parameter-varying unobservability subspaces containing a given subspace $\mathcal{L}$ is closed under subspace intersection. The minimal element of this family can be computed as the result of the parameter-varying $\mathcal{U}$ nobservability $\mathcal{S}$ ubspace $\mathcal{A l g o r i t h m}$ that can be obtained by duality from $\mathcal{C S A}$, for details see [27]. These subspaces were used in the solution of fault detection filter design problems. Stabilization issues were treated using LMI techniques; for details see [3] and [27]. Moreover, using these parameter varying unobservability subspaces it is also possible to solve fault detection filter design problems for qLPV systems; for details, see [5].

In [13], exactly the same algorithms were obtained in the context of bilinear systems.

\section{CONCLUSION}

This note investigated the extension of invariant subspace concepts used in the geometric theory of LTI systems to LPV and qLPV systems. The basic concept was the construction of subspaces invariant for a family of linear mappings. These were called parameter varying invariant subspaces. For LPV systems affine in their parameters algorithms were given to compute the relevant invariant subspaces used in relation with state feedback and observer design problems like DDP, decoupling, inversion, or detection filter design. These algorithms use only linear algebraic tools and can offer a computational alternative to some nonlinear problems reformulated in LPV or qLPV terminology.

\section{REFERENCES}

[1] P. Apkarian, P. Gahinet, and G. Becker, "Self-scheduled $\mathbb{H}_{\infty}$ control of linear parameter varying systems: a design example," Automatica, vol. 31, no. 9, pp. 1251-1261, 1995.

[2] G. Balas and J. Bokor, "Detection filter design for LPV systems," in Preprints of SAFEPROCESS'2000, vol. 2, Budapest, Hungary, 2000, pp. 653-656.

[3] G. Balas, J. Bokor, and Z. Szabó, "Failure detection for LPV systems-a geometric approach," in Proc. Amer. Control Conf., Anchorage, AK, 2002, pp. 4421-4426.

[4] J. Barker and G. J. Balas, "Flight control of a tailless aircraft via linear parameter-varying techniques," presented at the AIAA Guidance, Navigation, Control Conf., Portland, OR, 1999.

[5] J. Bokor, Z. Szabó, and G. Stikkel, "Failure detection for quasi LPV systems," presented at the 41 st IEEE Conf. Decision Control, Las Vegas, NV, 2002.

[6] G. Basile and G. Marro, "Controlled and conditioned invariant subspaces in linear system theory," J. Optim. Theory Applicat., vol. 3, pp. 306-315, 1969

[7] - "On the robust controlled invariant," Syst. Control Lett., vol. 9, pp. 191-195, 1987. 
[8] G. Becker and A. Packard, "Robust performance of linear, parametrically varying systems using parametrically-dependent linear dynamic feedback," Syst. Contr. Lett., vol. 23, pp. 205-215, 1994.

[9] S. P. Bhattacharyya, "Generalized controllability (A,B)-invariant subspaces and parameter invariant control," SIAM J. Alg. Disc. Meth., vol. 4, pp. 529-533, 1983.

[10] G. Conte, A. M. Perdon, and G. Marro, "Computing the maximum robust controlled invariant subspace," Syst. Control Lett., vol. 17, pp. 131-135, 1991.

[11] I. J. Fiahlo and G. J. Balas, "Design of nonlinear controllers for active vehicle suspensions using parameter-varying control synthesis," Vehicle Syst. Dyna., vol. 33, no. 5, pp. 351-370, 2000.

[12] P. Gahinet, P. Apkarian, and M. Chilali, "Affine parameter dependent Lyapunov functions and real parameter uncertainity," IEEE Trans. Automat. Contr., vol. 41, pp. 436-442, Mar. 1996.

[13] H. Hammouri, M. Kinnaert, and E. H. El Yaagoubi, "Observer-based approach to fault detection and isolation for nonlinear systems," IEEE Trans. Automat. Contr., vol. 44, pp. 1879-1884, Oct. 1999.

[14] R. Hermann and A. J. Krener, "Nonlinear controllability and observability," IEEE Trans. Automat. Contr, vol. AC-22, pp. 728-740, Oct. 1977.

[15] A. Isidori, Nonlinear Control Systems. New York: Springer-Verlag, 1989.

[16] P. Kabore, H. Hammouri, and M. Kinnaert, "A geometric approach to fault detection and isolation for bilinear systems: application to heat exchangers," in Preprints of SAFEPROCESS'2000, vol. 1, Budapest, Hungary, 2000, pp. 244-249.

[17] R. E. Kalman, "Contributions to the theory of optimal control," Boletin de la Sociedad Matematica Mexicana, vol. 5, pp. 102-119, 1960.

[18] A. Marcos and G. Balas, "Linear parameter varying modeling of the Boeing 747-100/200 longitudinal motion," presented at the AIAA Guidance, Navigation Control Conf., 2001.

[19] M. A. Massoumnia, "A geometric approach to the synthesys of failure detection filters," IEEE Trans. Automat. Contr., vol. AC-31, pp. 839-846, Oct. 1986.

[20] M. A. Massoumnia, G. C. Verghese, and A. S. Willsky, "Failure detection and identification," IEEE Trans. Automat. Contr., vol. 34, pp. 316-321, Mar. 1989.

[21] H. Nijmeijer and A. J. van der Schaft, Nonlinear Dynamical Control Systems. New York: Springer-Verlag, 1991.

[22] N. Otsuka, "Generalized invariant subspaces and parameter insensitive disturbance-rejection problems with static output feedback," IEEE Trans. Automat. Contr., vol. 45, pp. 1691-1697, Aug. 2000

[23] A. Packard and G. Becker, "Quadratic stabilization of parametrically dependent linear systems using parametrically dependent linear feedback," Adv. Robust Nonlinear Control Syst., vol. 43, pp. 29-36, 1992.

[24] C. De Persis and A. Isidori, "On the observability codistributions of a nonlinear systems," Syst. Control Lett., vol. 40, pp. 297-304, 2000.

[25] —- "A geometric approach to nonlinear fault detection and isolation," IEEE Trans. Automat. Contr., vol. 46, pp. 853-865, June 2001.

[26] L. M. Silverman and H. E. Medows, "Controllability and observability in time-variable linear systems," SIAM J. Control, vol. 5, pp. 64-73, 1967.

[27] Z. Szabó, J. Bokor, and G. Balas, "Detection filter design for LPV systems-a geometric approach," presented at the 15th IFAC World Congr., Barcelona, Spain, 2002.

[28] F. Szigeti, "A differential algebraic condition for controllability and observability of time varying linear systems," in Proc. 31st IEEE Conf. Decision Control, Tucson, AZ, 1992, pp. 3088-3090.

[29] F. Szigeti, J. Bokor, and A. Edelmayer, "On the reachability subspaces of time varying linear systems," in Proc. 3rd Euro. Control Conf., Rome, Italy, 1995, pp. 2980-2985.

[30] X. D. Sun and I. Postlethwaite, "Affine LPV modeling and its use in gain-scheduled helicopter control," in Proc. UKACC Int. Conf. Control, Sept. 1998, pp. 1504-1509.

[31] G. Stikkel, J. Bokor, and Z. Szabó, "Disturbance decoupling problem with stability for LPV systems," presented at the Euro. Control Conf., Cambridge, U.K., 2003.

[32] W. M. Wonham, Linear Multivariable Control-A Geometric Approach, 3rd ed. New York: Springer-Verlag, 1985

\section{Robust PI Controller Design Satisfying Sensitivity and Uncertainty Specifications}

\author{
Oded Yaniv and Mark Nagurka
}

\begin{abstract}
This note presents a control design method for determining proportional-integral-type controllers satisfying specifications on gain margin, phase margin, and an upper bound on the (complementary) sensitivity for a finite set of plants. The approach can be applied to plants that are stable or unstable, plants given by a model or measured data, and plants of any order, including plants with delays. The algorithm is efficient and fast, and as such can be used in near real-time to determine controller parameters (for online modification of the plant model including its uncertainty and/or the specifications). The method gives an optimal controller for a practical definition of optimality. Furthermore, it enables the graphical portrayal of design tradeoffs in a single plot, highlighting the effects of the gain margin, complementary sensitivity bound, low frequency sensitivity and high frequency sensor noise amplification.
\end{abstract}

Index Terms-Design, gain and phase margin, linear systems, proportional-integral (PI) control, robustness.

\section{INTRODUCTION}

Although many methods for tuning proportional-integral (PI) and proportional-integral-derivative (PID) controllers exist, extensive research in design techniques continues, driven by the strong use of such controllers in industry. Depending on the types of specifications that the design must satisfy, the tuning methods reported in the literature can be summarized as falling into one of two categories.

One class of methods considers gain and phase margin specifications. Ho et al. [1], [2] developed simple analytical formulae to tune PI and PID controllers for commonly used first-order and second-order plus dead-time plant models to meet gain and phase margin specifications. Ho et al. [3], [4] reported tuning formulae for the design of PID controllers that satisfy both robustness and performance requirements. Crowe and Johnson [5] presented an automatic PI control design algorithm to satisfy gain and phase margin based on a converging algorithm. Suchomoski [6] developed a tuning method for PI and PID controllers that can shape the nominal stability, transient performance, and control signal to meet gain and phase margins.

A second class of design methods focuses on sensitivity specifications, and is based on the premise that gain and phase margin specifications may fail to guarantee a reasonable bound on the sensitivity. Ogawa [7] used the QFT-framework to propose a PI design technique that satisfies a bound on the sensitivity for an uncertain plant. Poulin and Pomerleau [8] developed a PI design methodology for integrating processes that bounds the maximum peak resonance of the closed loop. The peak resonance constraint is equivalent to bounding the complementary sensitivity, which can be converted to bounding the sensitivity. Cavicchi [9] described a design method for

Manuscript received April 10, 2003revised May 29, 2003. Recommended by Associate Editor D. E. Miller. The work of M. Nagurka was supported by a Fulbright Scholarship for the 2001-2002 academic year allowing him to pursue this research in the Department of Computer Science and Applied Mathematics at The Weizmann Institute of Science (Rehovot, Israel).

O. Yaniv is with the Faculty of Engineering, Department of Electrical Engineering Systems, Tel Aviv University, Tel Aviv 69978, Israel (e-mail:yaniv@eng.tau.ac.il).

M. Nagurka is with the Department of Mechanical and Industrial Engineering, Marquette University, Milwaukee, WI 53201 USA (e-mail mark.nagurka@marquette.edu)

Digital Object Identifier 10.1109/TAC.2003.819646 